Int. J. Dev. Biol. 50: 223-232 (2006)

doi: $10.1387 / \mathrm{ijdb} .052051 \mathrm{cf}$

\title{
Morphogenesis, plasticity and irreversibility
}

\author{
CHIKARA FURUSAWA ${ }^{1,3, *}$ and KUNIHIKO KANEKO ${ }^{2,3}$ \\ ${ }^{1}$ Department of Bioinformatics Engineering, Graduate School of Information Science and Technology, Osaka University, Osaka, \\ ${ }^{2}$ Department of Pure and Applied Sciences, Univ. of Tokyo Komaba, Meguro-ku, Tokyo and \\ ${ }^{3}$ ERATO Complex Systems Biology Project, JST, Komaba, Meguro-ku, Tokyo, Japan
}

\begin{abstract}
The dynamical systems theory of morphogenesis is surveyed, in which positional information is generated through intracellular reaction dynamics and cell-cell interaction. Cells differentiate because of these dynamics. In addition, these differentiated cells form an ordered spatial pattern, which further stabilizes the cellular states, leading to robust morphogenesis and irreversibility in the differentiation. Induction, community effect, gastrulation and activin-controlled artificial tissue-genesis are discussed from the perspective of this theory and the relevance of dynamics of cellular plasticity is stressed.
\end{abstract}

KEY WORDS: morphogenesis, plasticity, irreversibility, evolution

\section{Question to be addressed: origin of positional informa- tion}

Biological pattern formation (morphogenesis) is currently understood as a change of genetic expression depending spatially on gradients of chemicals (Alberts et al., 2002). During morphogenesis, cells differentiate using developmental processes and these differentiated cells form an ordered spatial pattern. Experimental studies have now clarified how changes in gene expression occur during differentiation and in the spatial ordering process. However, a description of genetic changes by itself is insufficient to fully explain morphogenesis, because whether some genes are 'on' or 'off' depends on the chemical state of a cell and this is in turn influenced by interactions with surrounding cells.

Note that the process of pattern formation during normal development is rather stable. Thus, possible fluctuations of molecules and their effects on cell behavior are robust during normal developmental processes (Kaneko and Yomo 1999; Lacalli and Harrison 1991). Almost identical final patterns are formed and the temporal course for each pattern is also repeated. Furthermore, experiments to perturb the process by external removal or addition of cells suggest that the cells somehow 'know' their position within the whole system of an ensemble of cells, so that the damage can be repaired. Following these observations, Wolpert proposed the concept of positional information, which states that the cell knows its position by concentrations of diffusible chemicals (Wolpert 1969).

There are some problems in this positional information idea. The first concerns the origin of positional information. If some chemical gradient is externally given, one can specify the position. However, how the chemical gradient itself is generated is not itself answered within the positional information theory. To form a gradient in chemicals, cells at each end have to behave differently. However, to establish such differentiation, the pre-existence of a gradient is required in the positional information theory. In other words, how polarity is generated spontaneously is not solved. Thus, it is desirable to set up a theory in which the formation of positional information and cell differentiation reinforce each other.

Of course, in some cases, the gradient is given as a condition from the mother and exists in the unfertilized egg. An example is the concentration of some proteins such as bicoid in Drosophila (Driever and Nüsselein-Volhard 1988), through which the patternformation processes at the first stage of development is believed to be controlled.

However, not all the axes for the pattern are set as a maternally inherited effect. In addition, in mammals some homogeneity among cells seems to persist up to the fourth mitotic divisions. Even in other organisms that are more tightly controlled by maternal gradients, such problems may exist. For example, even though the initial concentration gradient of bicoid in the Drosophila egg may be changed experimentally, the final pattern of the entire body is robust with respect to this perturbation, up to a certain degree (Driever and Nüsselein-Volhard 1988).

This means that positional information is not always embedded in the initial condition of developmental processes, but is generated during them.

The second problem involves the robustness of morphogenesis. As a standard picture of developmental biology, cell differentiation progresses depending on the concentration of signal molecules. Because this is determined spatially, the cell state changes according to its position. In this way, a cell is believed to

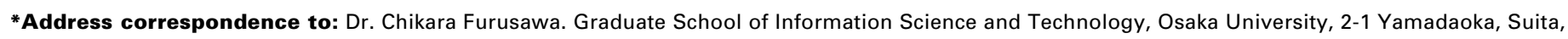
Osaka 565-0871, Japan. Fax: +81-6-6879-7432. e-mail: furusawa@ist.osaka-u.ac.jp 


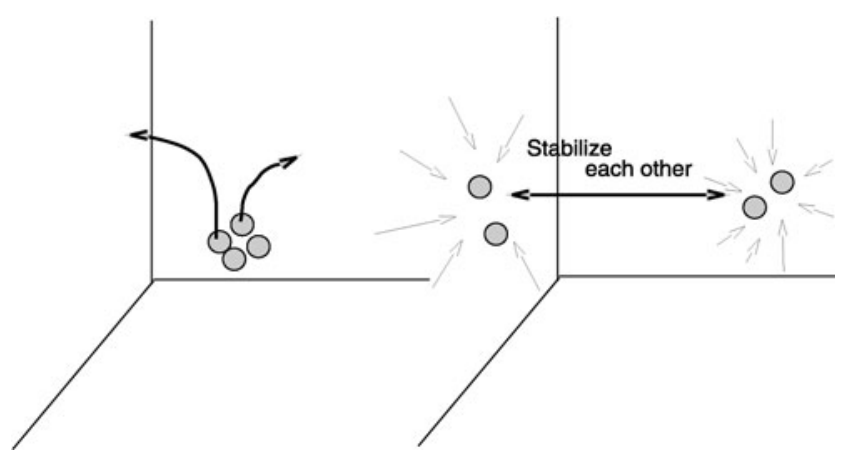

Fig. 1. Schematic representation of state space and differentiation. A homogeneous cell state is destabilized and, by taking distinct states, the cells mutually stabilize the states of the others.

'read' its own positional information. However, there may be large fluctuations in the chemical concentrations, which may cause a serious problem.

Recall that these signal molecules work often at very low concentrations. The quantity of chemical 'concentration' of relevance is given by the number of molecules per unit volume around the cell. Hence, the absolute numbers of signal molecules associated with cell differentiation are often small and in diffusion processes, each molecule moves randomly. Thus, there can be large fluctuations in the concentrations of molecules affecting a single cell in general. In fact, Houchmandzadeh et al. (2002) have demonstrated such large fluctuations in bicoid concentrations in Drosophila eggs at the same developmental stage. Such large fluctuations no longer appear at later steps in the developmental process and robust pattern formation results. Accordingly the threshold mechanism by itself is vulnerable under such fluctuation and cannot resolve the errors arising.

Before positional information theory, Turing proposed a theory in which spatial inhomogeneity of diffusible chemical concentrations emerges spontaneously (Turing 1952). In fact, the term 'morphogen' was introduced by Turing. In that pioneering study, Turing introduced a system of coupled elements interacting diffusively with each other. He showed that a spatial pattern is formed concerning the chemical concentration, starting from a'spatially homogeneous initial condition.

To be specific, consider two reacting chemicals: one that activates the reaction ('activator') and one that inhibits ('inhibitor'). When the diffusion constant of the activator is faster than that of the inhibitor, under certain conditions a homogeneous state with a constant concentration of the chemical is destabilized. Because of the instability of the homogeneous state, a pattern with some wavelength is formed, which then stabilizes the system. Indeed, Turing discussed pattern formation in a generalized diffusionreaction system. There are cases in which such instability leads to temporal rhythms. With more than two chemicals, there is also a case with spatiotemporal pattern dynamics, including spatiotemporal chaos. Although Turing's theory has not yet fully been appreciated in developmental biology, it is fully appreciated by those studying the physics and chemistry of non-equilibrium systems.

This theory partially answered the first question for positional information, by using dynamic instability and diffusive interaction. However, despite Turing's pioneering work on morphogenesis, some basic problems remain unsolved, i.e., on the origin and the robustness of the positional information. Furthermore, Turing's theory was not taken seriously by the biological community, because the genetic control mechanism discovered since then was thought to be essential.

First, although a periodic stripe pattern with alternation of chemical concentrations is generated by a Turing pattern, how the difference in concentrations leads to cell differentiation to 'discrete' cell types is not yet solved. Indeed, the change of chemical concentrations is rather continuous and it is not clear how discrete types represented by 'on' or 'off' states of genetic expression emerge from it. As differentiation is not well defined, the irreversible loss of potentiality is not studied either in Turing's theory.

Second, in the standard Turing model, developmental processes with the increase of cell numbers are not considered. In his original model, the system size (i.e. the number of cells) is fixed in time. Hence, neither the robustness nor the irreversibility of the developmental process is discussed.

During development, the initial few cells have the potential to differentiate to all other cell types: 'totipotency'. As development progresses, some cells lose this potential with time and in normal development this loss is irreversible. This irreversible loss of potential is influenced by interactions with surrounding cells and is related to the pattern formed by cells. Indeed, potentiality generally depends on what type of tissue of which a cell forms a part. For some tissues, when some cells are externally removed from the patter, regeneration can occur. However, for some other tissues, cells are already determined in their type and such regeneration is not possible. How such irreversible loss of potentiality for differentiation is related to morphogenesis is not yet answered.

The third problem concerns the discovery of gene regulatory mechanisms made after the publication of the Turing's paper. For example, in Drosophila, each segment corresponds to an expres-
A

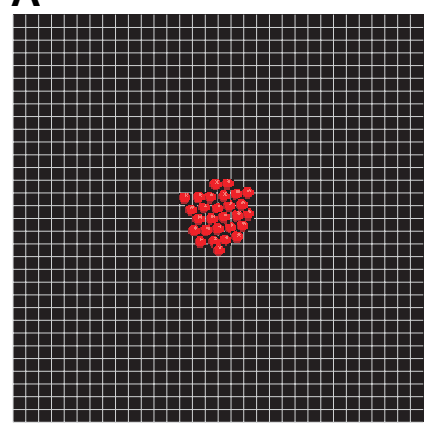

C

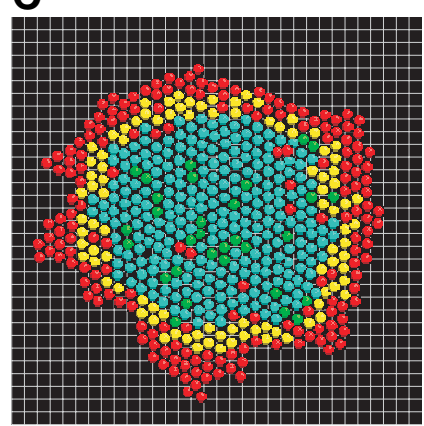

B

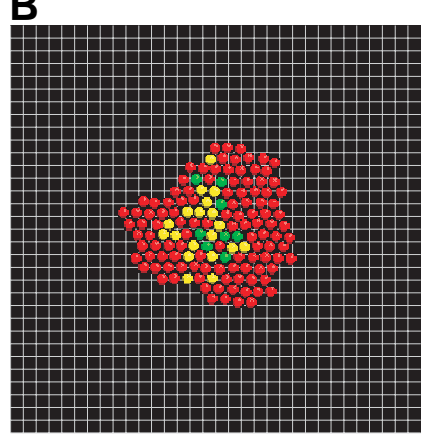

Fig. 2. Development of a cell cluster toward a ring pattern in a two-dimensional medium. Each symbol corresponds to a particular cell type, with different cell types distinguished by significantly different internal dynamics. Adopted from Furusawa and Kaneko 1998b, with permission. 
sion of some gene. From the perspective of gene regulatory dynamics, gene expressions are 'on' or 'off' according to the concentration of some signal molecules. Hence Turing's mechanism has not been considered seriously. Still, these observations do not necessarily contradict Turing's idea itself. If the idea is generalized, one can say that the interplay between intracellular reaction dynamics and the diffusion of chemicals leads to morphogenesis. This generalized viewpoint is consistent with the present molecular biology view based on the interplay between intracellular gene regulatory dynamics and diffusive signal molecules. What we need to add to the original Turing mechanism is sufficient complexity in the intracellular reaction dynamics. This is another missing link between Turing's and gene-regulatory network dynamics.

Despite extensive studies in Turing patterns (Meinhardt and Gierer 2000), the above three problems are not yet completely resolved. Previously, we proposed a theory to overcome such drawbacks and to explain the spontaneous differentiation of cells to form discrete types along with the increase in cell numbers. In the theory so far, we have disregarded spatial factors for simplicity. By including spatially local processes, it is possible to study how the dynamic differentiation process and spatial pattern formation are mutually reinforced and form stable patterns and cell types. Here we discuss these problems by reviewing some of our earlier studies.

\section{Logic}

In a cell, there are several chemical components and each cellular state depends on particular chemical compositions within a cell. These chemicals include proteins, RNAs, membranes and others. Through chemical reactions, the composition of a cell can change in time. With intracellular reactions, some chemicals are converted to products and at some stage the cell becomes large enough to divide into two. Without assuming any sophisticated programs, the two divided cells take almost identical chemical compositions, although there are some differences caused by random molecular fluctuations.

Note that there is positive feedback in the intracellular reactions. With nonlinear dynamics in the reaction, any tiny differences between the two cells may be amplified. This amplification depends also on cell-cell interactions, including competition for chemical resources for cell growth. As the cell number is increased, this cell-cell interaction is stronger and the amplification of tiny differences will be stronger. At some stage of cell numbers, this amplification becomes large enough to make a macroscopic difference between the two cell types. Now, the homogeneous states of a cell ensemble will be unstable.

On the other hand, these cells interact with each other through diffusion of chemicals across their membranes. Now through unstable dynamics, the cell state changes in time, also depending on the states of other cells. Here, it is expected that cells with similar states will compete strongly for resources for growth, whereas if they take rather different states, these cells will not compete with each other as much. It may then be expected that differentiation of cells comes to a stage that different types of cells mutually stabilize others' states. This mutual stabilization is possible under suitable cell-cell interactions (see Fig. 1 schematically).
A
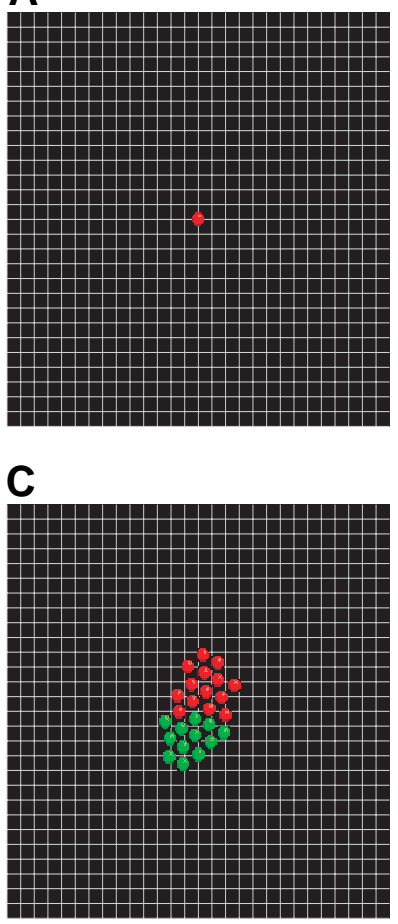

B

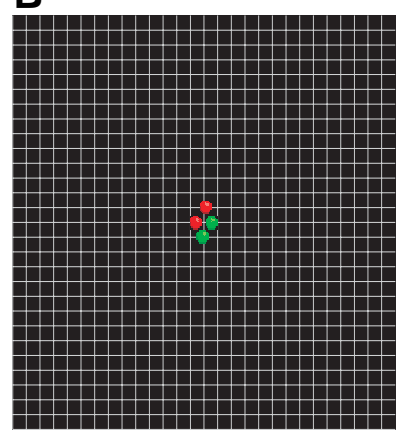

D

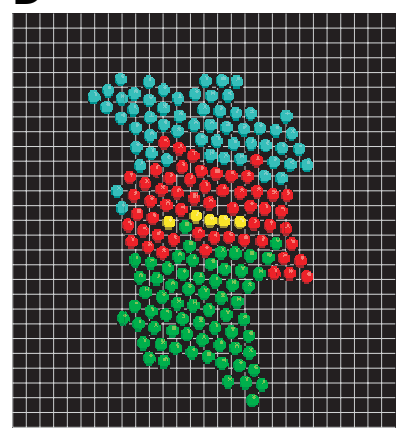

Fig. 3. Development of a cell cluster toward a striped pattern in a twodimensional medium. Each symbol corresponds to a particular cell type, distinguished by its particular type of internal dynamics, as shown in Fig. 4. From the stem-type cell S (red cell), the type A (green cell) differentiates and forms a cluster as shown in (C). With further increases in the number of cells, another type of cell, B, differentiates and forms a cluster at the upper side. With these processes, a stripe pattern forms (D).

This diversification of cells into a discrete set of types from a single cell type is a general consequence of interacting cells with biochemical networks and cell divisions, as was confirmed by several model simulations (Kaneko and Yomo 1994, 1997, 1999; Furusawa and Kaneko 1998a, 1998b) and based on dynamical systems theory (Kaneko 1990). According to the theory, differentiation proceeds first by loss of synchrony of intracellular oscillations as the number of cells increases. Then, the chemical composition of the cells is differentiated. The differentiated compositions become inherited by the next generation and such cell types are then determined. As a result of successive occurrence of the cell differentiation, the cell society will become composed of different cell types. This mechanism leading to differentiation is called 'isologous diversification', as it gives a mechanism by which an identical state (cell) can be diversified through the interplay between internal (reaction) dynamics and interactions. With this mechanism, cell differentiation is shown to be stable against molecular and other external fluctuations, where amplification of noise-induced slight differences between cells leads to a noise-tolerant society with differentiated cell types.

Now, to discuss morphogenesis, we need to consider also the spatial structure of these differentiated cell types. For this, we take into account the spatial locations of cells and the diffusion of chemicals through the space. Then, it is natural that the differentiated cell types by the mechanism discussed above are organized spatially to form a pattern that is robust against perturbation. 
With this pattern formation, gradients of chemicals are formed that consolidate the differentiation of cell types. Here, cell differentiation by intracellular dynamics leads to a gradient of chemicals and forms positional information, which strengthens the differentiation of internal states. With this reinforcement, differentiation by inter-and intracellular dynamics is transferred to a spatial pattern and differentiation is further stabilized. Cell differentiation is thus consolidated with the spatial pattern formation. Interplay among internal dynamics, intercellular diffusion of chemicals, cell division process and spatial pattern formation leads to such consolidation.

\section{Model}

Cells consisting of several chemicals are assumed to be located in space and chemicals diffuse through the space so that closer cells interact more strongly as a result. Chemicals that are permeable from the membrane diffuse through the space with a given diffusion constant and nutrients are supplied with a given diffusion rate from the outside.

(i) Internal chemical reaction dynamics: Cells are assumed to be surrounded by a two-dimensional medium that includes diffusive chemical substances. To represent cellular reaction dynamics, we assume that each cellular state is represented by concentrations of $k$ chemicals. Thanks to chemical reactions, the concentrations change over time. For the reaction dynamics, we choose a catalytic network among the $k$ chemicals. Each reaction from some chemical ito another chemical $j$ is assumed to be catalyzed by a third chemical $f$, these are all determined randomly. These reactions form a complicated reaction network. Here we simply choose randomly connected reaction networks and study common features in such system.

(ii) Cell - cell interaction: Cells interact with each other through the transport of some chemicals into and out of the surrounding medium. Here we consider only indirect cell-cell interactions via diffusive chemical substances, as a minimal form of interaction. We assume that the rates of chemicals transported into a cell are proportional to differences in chemical concentrations between the inside and the outside of the cell.

(iii) Cell division: Each cell receives penetrating chemicals from the medium as nutrients and reactions in the cell transform them into non-penetrating chemicals, which form the body of the cell. From these reactions, the amount of chemicals in each cell changes. The chemical compositions of the two divided cells are almost identical (except for some molecular fluctuations). Cells have a given size within this space and when a cell divides, its
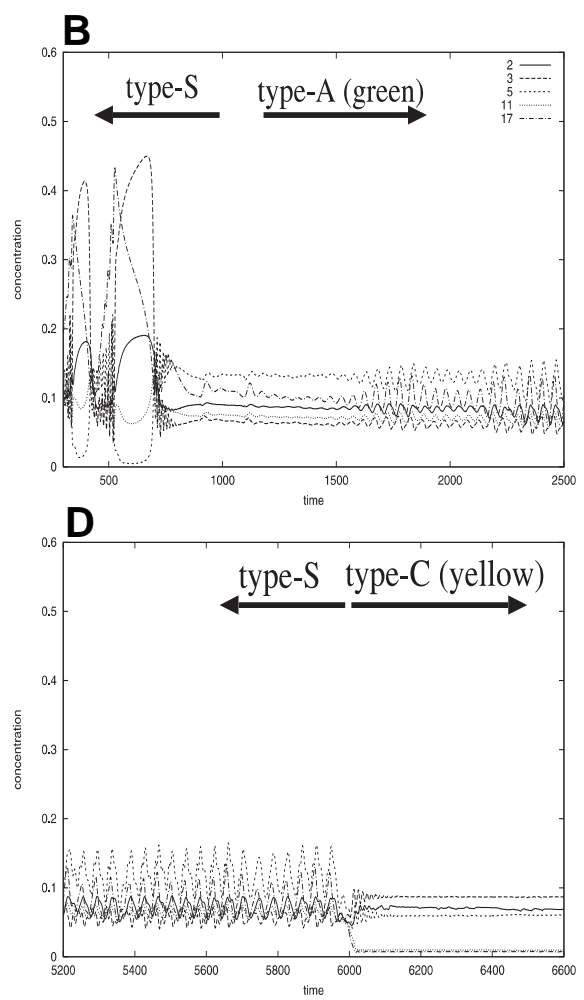

Fig. 4. Time series of concentrations in the example of a striped pattern. (A) Time series of as a function of time. In this Figure, we have plotted the time series of only six of the 32 internal chemicals, for clarity. (B-D) Time series of concentrations in a cell, representing the course of differentiation to type $A, B$ and $C$ cells, respectively.

offspring cell is located in the neighborhood of the original cell. The directions for placing the sibling cells are chosen randomly.

For details of the model, see Furusawa and Kaneko (1998a, 1998b, 2002, 2003). ${ }^{1}$

\section{Results}

In this case, as the number of cells increases, first the cells start to differentiate, as discussed in previous chapters. The different cell types take different chemical compositions. Together with this process of differentiation, these different types of cells start to form some spatial pattern. Here, first intracellular reaction dynamics and cell-cell interactions lead to cell differentiation, which later establishes spatial pattern.

We discuss two examples for the development in a twodimensional space. In the example of Fig. 2, from the initial stem type $S$ (represented by a red cell in Fig. 2), type $X$ (yellow) and type $Y$ (green) cells form through differentiation at the inside of the circle. The concentric circle pattern with type $X, Y$ cells inside is formed first. As these cells proliferate, there appears a new type of cell $Z$ (blue) inside the cluster of type $X$ cells, by differentiation from type $X$ cells. These cell types again take distinct chemical compositions. The type $S$ cells have greater chemical diversity

Note 1: As the initial state, a single cell, whose chemical concentrations are determined randomly, is placed in the medium. According to the process described above, cells divide to form a cluster. With the increase in the number and volume of cells, chemicals in the medium are consumed. To maintain the growth of the organism, the system is in contact with a bath of chemicals from which nutritive chemicals are supplied. 
and variation. Here successive differentiation $(S \rightarrow S, X, Y),(X \rightarrow X, Z),(Y \rightarrow Y),(Z \rightarrow$ $Z$ ) can be observed.

The second example is of stripe formation. In this case, a single cell placed at the center of the medium exhibits oscillatory reaction dynamics and has high chemical diversity (Fig. 4A). There, type A (green) cells first differentiate from the original type $\mathrm{S}$ (red) cells at one side, as shown in Fig. 3B. Along a specific axis, $S$ and $A$ are differentiated. At this stage the differentiation rule is given by $(S \rightarrow S, A)$ and $(A \rightarrow A)$. As the numbers increase further, the state of type $S$ cells at the other side of $A$ is destabilized, to differentiate to a new type $B$ (blue). Hence, the stripe pattern is formed with $\mathrm{A}, \mathrm{S}, \mathrm{B}$. Here the differentiation rules are $(S \rightarrow S, A, B),(A$ $\rightarrow A)$ and $(B \rightarrow B)$. From these differentiations, a spatial pattern of cells consisting of three stripes, each containing cells of just one kind, is formed, as shown in Fig. 3D. Later, another type $C$ is differentiated from type $S$ near the border of $S$ and $A$. The intracellular dynamics of each cell type are shown in Fig. 4.

In a system exhibiting the stripe pattern, point symmetry is broken, in contrast to the ring pattern (Fig. 2C). This is because of the development that takes place at the beginning of the differentiation process from type $S$ to type $A$ cells when there is still only a few cells (Fig. 3B). It is at this stage that point symmetry is lost and this asymmetric small cluster of cells expands through further cell divisions. Because of the difference regarding both rates and types of nutrients absorbed and released by type $S$ and type A cells, the asymmetric distribution of cells brings about asymmetric concentration gradients of nutrients in the medium, as shown later.

\section{Generation of positional information}

Now we discuss how positional information is generated with this differentiation process. Before going into specific discussion, we recall again the requisite for 'information'. As discussed in Kaneko and Yomo (2002), the information carrier has to satisfy (1) controllability and (2) preservation. If a given chemical concentration gradient works as positional information, we need to discuss (i) how it is generated from dynamics,(ii) how it controls the differentiation of cells and (iii) how such a gradient is preserved under molecular fluctuations and external disturbances.

We focus on the example leading to a stripe pattern. In Fig. 5, we have plotted the concentration of resource chemicals along the long axis of this cell aggregate. Despite the constant boundary condition to supply this chemical, a chemical gradient arises. Here the concentration gradient and the region of each type of cell has some relation. For example, at the region of the type $S$ and type $B$ cells the resource chemical 0 has a higher concentration and at the region of type $A$ cells a higher concentration of chemical 1 is maintained.

Conversely, if we place a type $S$ cell in a region with a higher concentration of chemical 1 (or 2), differentiation to type A (or B) follows. In this sense, the gradients of these chemicals serve to
B

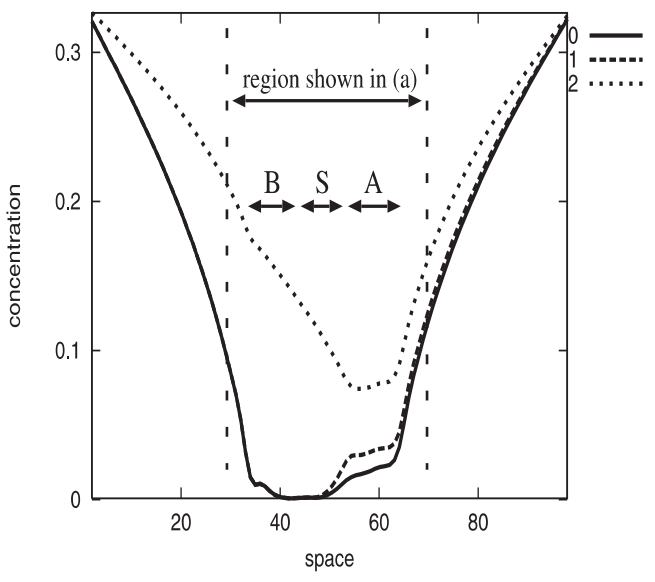

ace

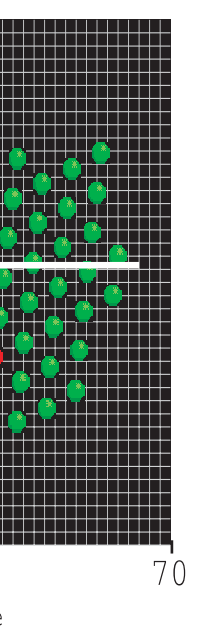

long th

ng the st

ripe pattern. In (B), concentrations of three chemicals in the medium are plotted as functions of position along the segment (a)' in (A). In (B), the regions of different cell types (i.e. regions of blue, red and green cells) are also shown by the arrows. At each end of the medium, the concentrations of all chemicals are fixed, because these chemicals are continuously supplied into the medium from a chemical bath at each end with fixed concentrations of chemicals.

control a differentiation of a cell into each type. Accordingly, the chemical concentrations are 'read' by each cell and act as its positional 'information'.

Depending on the concentration of the particular chemical environment for each cell, the cell state will change slightly, even if it belongs to the same type. Although differentiation into types is discrete, there arises slight modulation of each cell's state depending on its position. In Fig. 6, we have plotted $\Sigma(x)^{2}$ of each cell along the axis, where $x$.indicates the intracellular concentration of the $j$ th chemical. One can first see clear discrete differentiation into types as the states of the cells of the same type are slightly modulated by their individual positions. This modulation (analogue difference) is well discernible for type $S$ and B cells. It is interesting to note that, besides the digital information on type, there is analogue information provided by cell position, which brings about a continuous modulation of the cell state. Note that, for the type A cells, this modulation is much smaller. We will return to this point later.

\section{Complementary relation between internal cell state and po- sitional information}

Once a pattern is formed together with the generation of positional information by a concentration gradient, both the pattern and information are stable against some perturbations, such as removal of some cells, or perturbations of chemical concentrations into some cells. After perturbations, both the pattern and chemical concentration gradient return to the original states because the cell state and gradient are mutually stabilized. Thus, the property required for preserving information is realized.

However, this does not necessarily mean that only the positional information is sufficient to produce the pattern. For example, if we randomize the cell state, by changing each intracellular concentration, while maintaining the environmental chemical gradient, then the subsequently generated cell pattern is quite disordered, as in Fig. 7. The original regular stripe pattern is totally 
A

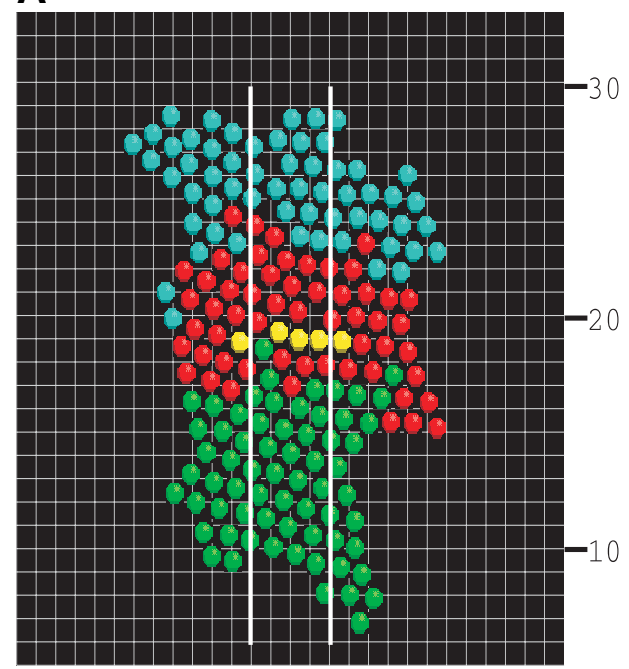

B

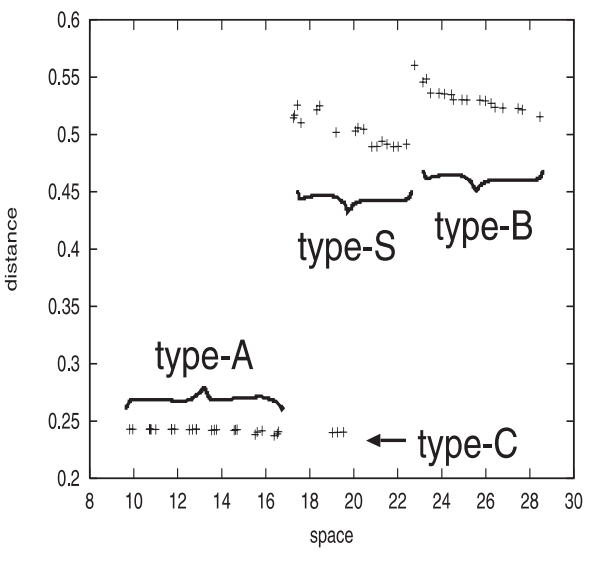

Fig. 6. Variation of intra-cellular dynamics with respect to position. To measure the change undergone by the intracellular dynamics when the position of a cell is changed, we determined the average position of the orbit of each cell in the k-dimensional phase space, which was calculated as the averages of the $k$ concentrations over a certain period for that cell. Each point in (B) corresponds to a cell placed between the two solid lines in (A), and the Euclidian distance in the phase space between the average position for a cell and the origin is plotted as a function of the position of the cell in the medium.

lost. Hence positional information alone is insufficient to regenerate the pattern. If the intracellular state is too different, the cell cannot read the information. Such a complementary interaction between the internal cell state and positional information is important.

\section{Regeneration process}

Now we discuss the problem of regeneration in relationship with irreversible differentiation. In the present model case of stripe pattern formation, the type A cell proliferates both the same type of cell and the type B. What happens if some cells are removed to destroy the field of original positional information? Does the cell

A

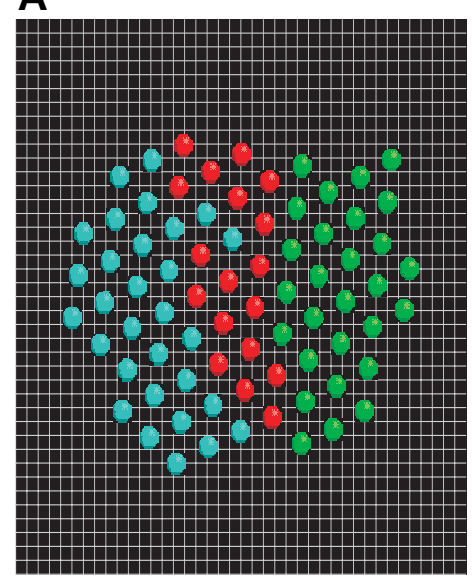

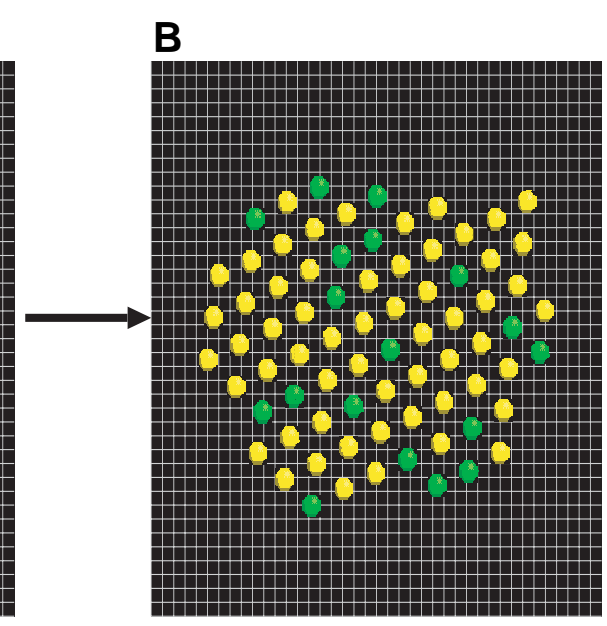

Fig. 7. Spatial patterns of cells when multiple cells, instead of a single cell, are placed in the generated positional information (i.e. chemical gradient). The initial chemical concentrations in the medium were set to be the same as in Fig. 5. Note that positional information alone is insufficient to reproduce the original pattern. ensemble have the potential for regeneration?

(I) Recovery from the removal of the entire type $B$ region: In one case, we removed all the type B cells (Fig. 8A), after the stripe pattern had developed. After this operation, the rate of transitions from type $S$ to type $B$ cells was enhanced at the side of type $S$ region farthest from the type $A$ region; as a result, the stripe pattern with three layers gradually re-appeared.

(II) Recovery following the removal of the entire type S region-de-differentiation of type $B$ cells into type $S$, induced by the interaction with type A cells: In this case, we removed all the type $S$ cells located in the middle of the stripe pattern and combined the remaining cell clusters consisting of only type $A$ and type $B$ cells, as shown in Fig. 8B. After this alteration, type 2 cells located at the boundary between the type $A$ and type $B$ regions de-differentiated back into type $S$ cells and the stripe pattern with three layers was thereby recovered. It is important to note that de-differentiation from a type $B$ cell to a type $S$ cell never occurs during the 'normal' course of development (i.e. without perturbation).

As shown, recovery is possible after major damage to the system. Via this damage, positional information by concentration gradient is also largely destroyed. However, positional information is regenerated through the recovery process. This is possible because the positional information is not given externally, but rather is generated through intracellular chemical reaction dynamics and cell-cell interactions.

(III) Formation of a new pattern resulting from the removal of the type A region: Here, all the type A cells were removed from a cluster with a striped pattern (Fig. $8 \mathrm{C}$ ). In this case, regeneration of the type A region was not observed. Instead, type $S$ cells at the periphery of the cluster differentiated into type B cells. As a result, a sandwich-like B-S-B structure formed and, with further development, a ring structure with inner type S cells and outer type B cells formed. In this case, the final cell society consisted only of type $S$ and type $B$ cells.

In this example, once a type B cell is formed, the differentiation from $S$ to $A$ is inhibited. In the 'normal' process, the type $A$ cell is already differentiated before the type B; the A-S-B pattern is generated and inhibition is ineffective. In the present case, without the existence of type A cells, the existing type B cells inhibit the differentiation into type A from type $S$. In this way, the ordering (or history) of the developmental process is important.

Why did type $B$ cells, rather than type A cells, differentiate when type $A$ and $B$ cells were attached? Here one should note that the variation of the chemical concentrations of type A cells is much smaller than the other types, as displayed in Fig. 6 and as discussed in 
the fluctuation-response relationship (Sato etal., 2003), the state with smaller fluctuations or variations has a smaller response against external changes and has a lower plasticity. The type B cells have larger fluctuations and variations against external changes than the type A cells. Hence, the type B cells have a higher plasticity and are more readily changed. Thus, they have a higher potential for de-differentiation when external conditions are varied.

\section{Importance of ordering in development}

As shown in the above examples, the history of how an ensemble of cells develops along with increasing cell numbers is essential to the selection of a pattern. The cells that are generated later are put in a field of chemical concentration that has already been produced. The selection of cell state is then determined accordingly. This point is not well discussed, at least in the original Turing pattern. To reexamine this issue, we put 100 cells initially in the configuration that was generated through the original simulation. Here the initial condition was set so that it has a homogeneous chemical concentration. The pattern thus formed and plotted has no regularity, as in Fig. 7. In the term of dynamical systems, the selection of initial and boundary conditions through development is essential to form an ordered pattern.

\section{A constructive experiment}

Here we discuss a constructive experiment to form a tissue from undifferentiated cells artificially. To be specific, we explain recent controlled tissue generation by Asashima's group (Ariizumi and Asashima 2001; Uochi and Asashima 1996). They removed some cells from the animal cap of Xenopus embryos. These cells were isolated from each other and then put into a solution of activin for a while. This ensemble of undifferentiated cells, was cultured to examine development. Here the initial cells were almost homogeneous. Surprisingly, merely by changing the concentration of activin, various frog tissues, including notochord, heart and muscle, were generated. Further, using a solution of retinoic acid and Conacanavalin $A$, they succeeded in forming over a dozen tissues, including nephrons and sensory organs such as eyes and ears. These results will be important for tissue engineering. Indeed, they have confirmed that such generated tissues are functional, by putting them into an adult body. Here, however, we discuss the importance of these results in considering the logic of development.

The following two points should be noted in relation to our theoretical results. They show that the naive picture of a threshold mechanism for development is insufficient. The first feature suggests the existence of attractors or attracting states as a dynamical system at a tissue level. The second feature suggests that differentiation is determined through an interplay between intracellular dynamics and cell-cell interaction, as adopted in our study.

(i) Jump-over-generation of tissues, jumping over normal temporal course of development: Activin concentration was adopted as a control parameter in the Asashima group's experiments. Activin is important in normal development, as Asashima had already discovered. However, the high concentration used in their experiments is not actually achieved in normal development. In addition, during normal development, several other molecules are involved as controllers. Hence, the experimental process they adopted progressed in a rather different situation from that in normal development. The most surprising outcome is that, despite this difference from the normal course, the final tissues generated were the same as those emerging from normal development. This was verified by the morphology of tissues, cell types and gene expression patterns. Normal function
A

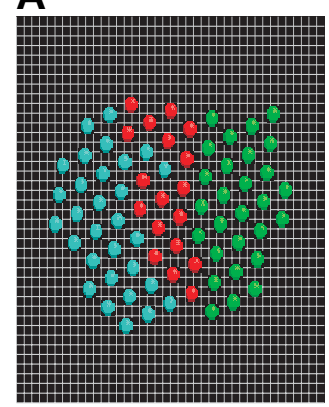

$\downarrow$
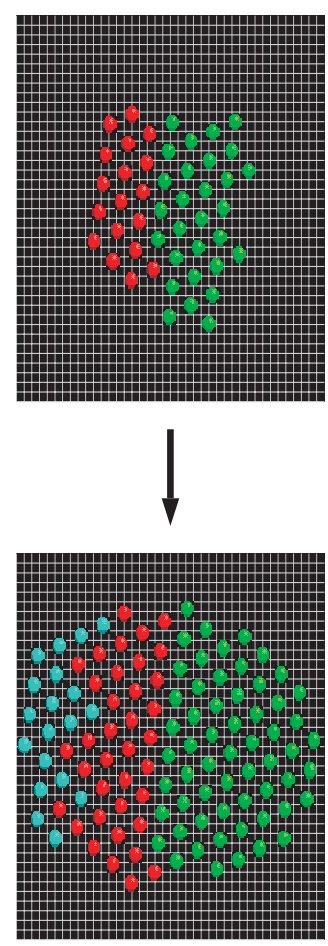

B
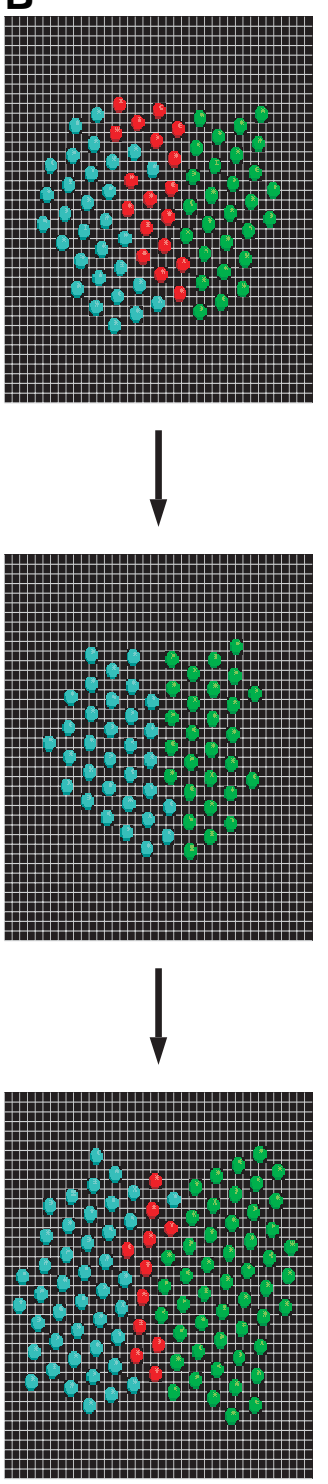

C
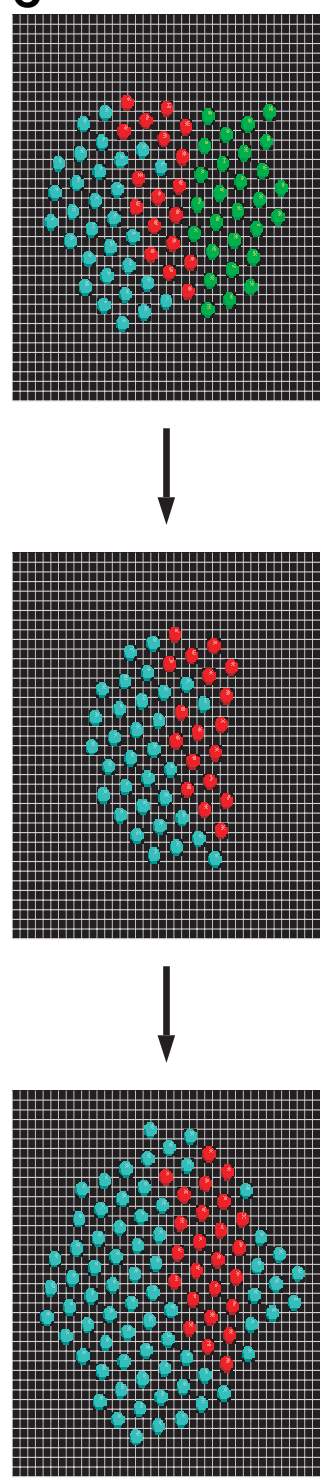

Fig. 8. Regeneration of the stripe pattern. (A) When the type $B$ cells are removed, they are differentiated from the type $S$ cells to reproduce the original stripe pattern. (B) When the type $S$ cells are removed, they are de-differentiated from the type $B$ cells to reproduce the original stripe pattern. (C). When the type A cells are removed, type $B$ cells are differentiated from the type $S$ cells to produce a novel $B-S-B$ pattern. Based on Furusawa and Kaneko 2003b. 
was confirmed by transplanting these constructed tissues, such as the heart or eye.

However, the 'process' to achieve these tissues is quite different from the normal case. During normal development, starting from undifferentiated cells, first mesoderm, including notochord and muscles, forms, from which neural systems are generated and later some sensory organs are formed. In these 'constructive experiments', without going into detail, the goal tissue was generated by jumping over processes that would normally form earlier tissues.

These results contest the viewpoint that developmental process should constitute a succession of finely tuned stepwise processes. Rather, it is more natural to adopt the viewpoint that the final tissue is a kind of 'attractor' in a very large dynamical system and there can be several paths to reach it other than via normal development. Of course, such paths to attractors are intermingled and complex. Obviously, the basis for such attractors needs some control. The results of Asashima's group suggest

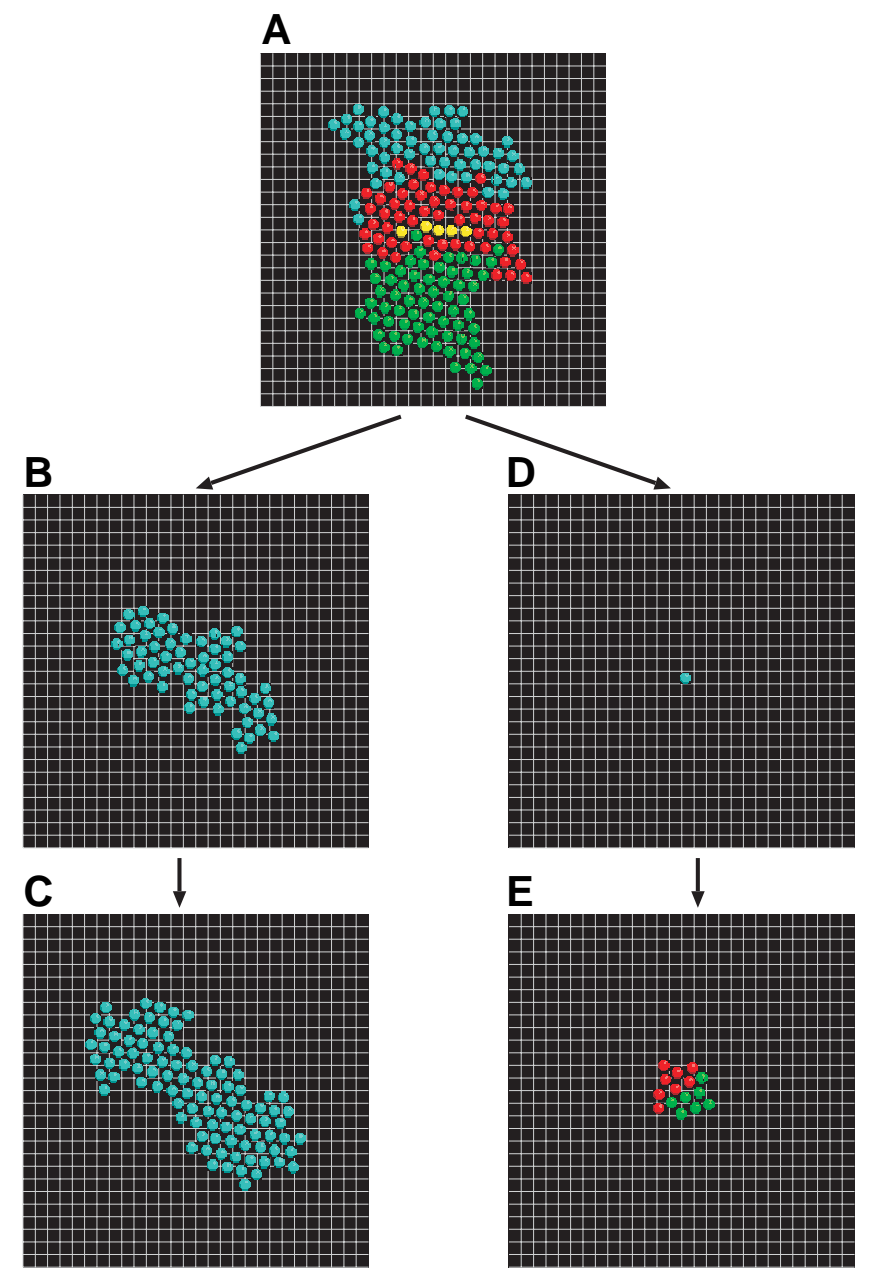

Fig. 9. The community effect in the case of a striped pattern. From an ensemble of cells exhibiting a striped pattern, a group of type B cells or a single type $B$ cell were transplanted into a new, otherwise unoccupied medium and they developed under the same rules and parameter values as the previous case. Type $B$ cells transplanted as a group remain type $B$ cells, whereas a type $B$ cell transplanted as a single cell transforms into a type $S$ cell and then eventually develops into a striped pattern. that the change of activin concentration they used was not a finely tuned control, but rather served to perturb the initial state by destabilizing the cellular state so that it moved toward a state of attraction to some other tissues.

(ii) Community effect - the formation of tissue is highly dependent on the number of cells: In the experiments of Asashima's group, a number of undifferentiated cells were taken from the animal cap of Xenopus embryos and with these the construction of several types of tissue (e.g. heart, notochord and muscle) was caused by controlling the concentration of activin. In these experiments, how the construction of tissues proceeded depended on the numbers of cells used. Indeed, normal tissues were generated only for a precise range of initial cell numbers. For pronephros formation by the solution of activin (with $10 \mathrm{ng} / \mathrm{mL}$ ) and retinoic acid, the normal tissue was formed only if the number of cells was around $300-500$. When there were fewer than 100 cells, the cells died, whereas when more than 500 cells were used, inhomogeneous tissues were generated. Construction of heart tissue by activin was also only possible for a limited range of cell numbers. This experimental result cannot be understood solely by signaling to one of the cells and cell-cell interaction is essential, as has been discussed in the previous section.

\section{Summary and Discussion: development as dynamics of plasticity}

\section{Summary}

We now summarize the logic of our theory and results of our simulations and discuss their relevance to biological morphogenesis.

In our theory, cells possessing internal nonlinear reaction dynamics differentiate into several distinct cell types when the cell number exceeds a threshold value. The transition from one cell type to another is regulated by the position of the cell in question. This regulation leads to an ordered spatial pattern consisting of differentiated cells, as shown in Figs. 2 and 3. Each cell 'reads' information from the external field and reacts to this information by modulating its intracellular dynamics in accordance. This modulation controls the rates of differentiation into various cell types, while such cell differentiations, in turn, alter the state of the environment. With this circular relationship between intra- and intercellular dynamics, the gradients of chemicals in the medium act as positional information controlling the fate of each cell. This interaction between intra- and intercellular dynamics is responsible for the robustness of the developmental process.

\section{Community effect}

Gurdon showed that cell commitment (i.e. determination to a certain cell type) depends on the total cell number and termed this cell-number effect as the 'community effect' (Gurdon, et al., 1993). When the number of cells is small, differentiation is not determined and the cell state changes in time; whereas when the cell number is larger, the cell state is mutually stabilized and is maintained. The experiments by Asashima's group also show clearly how tissue formation is critically dependent on cell numbers.

Because our theory is based on cell-cell interactions, a 'community effect' is to be expected. Indeed, we have studied this problem by taking the stripe case. For example, when a single 
type B cell (such as that in Fig. 3) is placed in a medium containing no other cells, this cell de-differentiates back into a type $S$ cell. However, as shown in Fig. 9, the type B cell state is stable when such a cell is surrounded by a sufficient number of other type $B$ cells. To confirm this point, we have carried out simulations with the same model, but placing a large number (more than 10) of type $B$ cells in an otherwise unoccupied medium. In this case, a cell colony consisting of only type B cells grows.

As discussed previously (Furusawa and Kaneko 1998), there can be multiple stable cell colony states. A cluster consisting only of type B cells corresponds to one such stable state, but this state never appears in the ordinary developmental process starting from a single cell. In this case, only when the number of type $B$ cells initially placed in the medium is more than around 15 do these cells remain in their type B states. Otherwise, all the cells de-differentiate into type $S$ cells. This clearly indicates that the states of the cells existing in a cluster can be mutually stabilized by their interactions.

\section{Induction and plasticity}

The term 'induction' is often adopted to describe development. This means that when some group of cells start to interact with another group, cells of one group change their state to form a different tissue. In fact, both cell groups interact mutually, but only one group of cells is changed, or 'induced' by the other group. This means that 'changeability' of the cell state is higher for the former than the latter group. This is nothing more than the problem of the degree of plasticity in cells.

We wonder that the 'plasticity' seen here might seem ambiguous. Still, in several models of cell differentiation (Furusawa and Kaneko 2000, 2001), we have observed empirically that plasticity can be quantitatively represented by the following measures.

(i) A cell with larger fluctuations or temporal variation of chemical concentrations (say via differential gene expression) has higher plasticity: i.e., its susceptibility to the external condition's changes is greater. Fluctuations and susceptibility (changeability) are therefore correlated according to the fluctuation-response relationship (Sato et al., 2003).

(ii) A cell with diverse chemicals has higher plasticity. In other words, those cells with weak but diverse gene expressions exhibit higher plasticity.

Hence, the concept of 'plasticity' can be measured experimentally by the current tools of experimental biology. Assuming a quantitative measure of plasticity, we propose the following hypothesis:

When two groups of cells with different plasticity meet, the group of cells with higher plasticity is changed more. In other words, the group with lower plasticity brings about the induction of the other group of cells with a higher plasticity.

Now we can check the validity of this hypothesis from our simulation. In the model we studied here, when the type $A$ and $B$ cells are put adjacent, the type $B$ cells de-differentiate and change to type $S$ cells, whereas when type $S$ and $B$ cells are adjacent, there is differentiation from type $S$ cells. The 'changeability' of cells is in the order of $S>B>A$. Now recall the data of variation of chemical concentrations among cell types. These variations and fluctuations of each cell type, again decrease in the order of $\mathrm{S}>\mathrm{B}>\mathrm{A}$. Hence, the hypothesis that the variation or fluctuation gives the degree of plasticity is confirmed. When there are two types of cells, generally those with higher plasticity are changed (induced) and this plasticity can be measured quantitatively as variation or fluctuation of cellular states.

\section{Transformation of state differentiation into spatial pattern}

It should be noted again that the pattern formation seen here is not predetermined from spatial information, but rather through intracellular dynamics and interaction. Spatial patterns and intracellular states mutually stabilize robust pattern formation consisting of several cell types. Here differentiation by intracellular dynamics is consolidated to a pattern.

Although we have here adopted oscillatory catalytic reaction dynamics for intracellular dynamics, the present mechanism does not necessarily require such dynamics. As long as there is some instability in intracellular dynamics, this mechanism is effective. Indeed, Takagi and Kaneko (2005) have shown that the present mechanism works starting from intracellular dynamics with multiple fixed points. Another choice for intracellular dynamics, that straightforwardly corresponds to gene regulatory dynamics, is the use of threshold dynamics so that each gene expression takes either high or low values, corresponding to 'on' or 'off' states of genes, respectively (Mjolness, Sharp and Reinitz 1991; SalazarCiudad, Garcia-Fernandez and Sole 2000). Even with these dynamics, there can be instability in the intracellular state(Ishihara and Kaneko 2004). Using such gene regulatory dynamics and applying the present scheme, it is shown that the present scenario for morphogenesis works. In this sense, the third question addressed to the Turing's mechanism - how dynamical systems mechanism and gene regulatory mechanisms are consistent - is answered.

We should mention here that Newman put forward the idea that Turing patterns will be consolidated to gene expression (Newman and Comper 1990; Newman 1994, 2003). The present theory is consistent with this idea.

\section{Destabilization of intracellular state and regain of plasticity; an interpretation of gastrulation}

In the development of vertebrates, there is an important epoch: gastrulation. As the number of cells increases beyond some threshold value, the cells start to move, triggered by mechanical instability such as buckling, so that some cells start to move inside the other group. Although the origin of gastrulation itself could be mechanical, an important consequence of this is that cells that were far apart and that did not interact with each other start to make contact directly and this novel cell - cell interaction leads to a drastic change in cellular states.

Now, the stability of the cell state is not determined solely by intracellular factors, but also through interaction with neighboring cells. Then, when cells start to contact some other cells that were originally far away, the cell state can be destabilized, which may lead to novel differentiation.

Note that the plasticity in a 'closed' system generally decreases (or does not change) over time. All of our simulation results of cell differentiation processes support this proposition. As long as the change of chemical states of cell follows their intracellular chemical reaction dynamics and cell-cell interaction in a given spatial configuration, plasticity will decrease with time. In the case of gastrulation, however, it seems that the plasticity is regained, following the destabilization of the cell state, as it is 
triggered by novel contacts of cells that were not initially adjacent. Here, mechanical instability of cell configurations leads to cell movement and novel arrangements of cells. Hence, a novel type of dynamic that was not included in the original chemical system sets in here. In this, sense the system is 'open' to a new dimension of interaction and allows for the recovery of plasticity.

Now we reconsider the experiments of Asashima's group from this viewpoint. We propose that, after cells are put in the solution of activin, the cell state must be destabilized to recover some degree of plasticity. Through this destabilization, a new path emerges to states that were hitherto inaccessible. As the concentration of activin is increased, a new path to a tissue that was hard to reach is opened. Now, in the process of gastrulation, contact with new cell aggregates will lead to a similar destabilization. Then, the longer the contact, the greater the instability and a developmental path to a tissue that was harder to reach should be opened. If the argument here is correct, the increase of contact time between cells that were initially separate in the course of gastrulation and the increase of the concentration of activin in the artificial development experiment must have similar effects. In fact, the ordering of notochord, pronephros, muscle, in the order of concentration of activin in the experiments by Asashima et al., corresponds exactly to the order of tissues generated during gastrulation. (here the cells that first make contact with new cells in gastrulation have a longer time of contact and then develop to form muscle). Although the discussion here is premature, it will be important in future to understand the relationship between plasticity of cellular states and the ordering of induction as well as the tissue formation by external control.

\section{Acknowledgments}

The authors would like to thank Makoto Asashima and Tetsuya Yomo for stimulating discussions.

\section{References}

ALBERTS, B., BRAY, D., LEWIS, J., RAFF, M., ROBERTS, K. and WATSON, J.D., The Molecular Biology of the Cell, 1983,1989,1994,2002

ARIIZUMI, T. and ASASHIMA, M. (2001) In vitro induction systems for analyses of amphibian organogenesis and body patterning. Int. J. Dev. Biol. 45, 273-9

DRIEVER, W., NÜSSELEIN-VOLHARD, C. (1988) A gradient of bicoid protein in Drosophila embryos. Cel/54, 83-93

FURUSAWA C. and KANEKO, K. (1998a) Emergence of rules in cell society: differentiation, hierarchy and stability. Bull. Math. Biol. 60 659-687

FURUSAWA C. and KANEKO, K. (1998b) Emergence of multicellular organisms with dynamic differentiation and spatial pattern. Artif. Life 4 78-89

FURUSAWA C. and KANEKO, K. (2000) Origin of complexity in multicellular organisms. Phys. Rev. Lett. 84 6130-6133

FURUSAWA C. and KANEKO, K. (2001) Theory of robustness of irreversible differentiation in a stem cell system: chaos hypothesis. J. Theor. Biol. 209 395-
416

FURUSAWA C. and KANEKO, K. (2002) Origin of multicellular organisms as an inevitable consequence of dynamical systems. Anat. Rec. 268 327-342

FURUSAWA C. and KANEKO, K. (2003) Robust development as a consequence of generated positional information. J. Theor. Biol. 224 413-435

GURDON, J.B., LEMAIRE., P. and KATO, K. (1993) Community effects and related phenomena in development. Cel/75 831-834

HOUCHMANDZADEH B., WIESCHAUS E., LEIBLER, S. (2002) Establishment of developmental precision and proportions in the early Drosophila embryo. Nature 415 798-802

ISHIHARA, S. and KANEKO, K. (2004) Magic number $7 \pm 2$ in networks of threshold dynamics. Phys. Rev. Lett. 94058102

KANEKO, K. (1990) Clustering, coding, switching, hierarchical ordering and control in network of chaotic elements, Physica 41 D 137-172

KANEKO, K. and YOMO, T. (1994) Cell division, differentiation and dynamic clustering, Physica 75 D, 89-102

KANEKO, K. and YOMO, T. (1997) Isologous diversification: a theory of cell differentiation. Bull. Math. Biol. 59 139-196

KANEKO, K. and YOMO, T. (1999) Isologous diversification for robust development of cell society. J. Theor. Biol. 199 243-256

KANEKO, K. and YOMO, T. (2002) On a kinetic origin of heredity: minority control in replicating molecules. J. Theor. Biol. 312 563-576

LACALLI, T.C. and HARRISON, L.G. (1991) (1991) From gradient to segments: models for pattern formation in early Drosophila embryogenesis. Sem. Dev. Biol. 2 107-117

MEINHARDT, H. and GIERER, A. (2000) Pattern formation by local self-activation and lateral inhibition. Bioessays 22 753-760

MJOLSNESS, E., SHARP, D. H. and REINITZ, J. (1991) A connectionist model of development. J. Theor. Biol. 152 429-453

NEWMAN, S.A. and COMPER, W.D. (1990) Generic physical mechanisms of morphogenesis and pattern formation. Development 110 1-18

NEWMAN, S.A., (1994) Generic physical mechanisms of tissue morphogenesis: a common basis for development and evolution. J. Evol. Biol. 7 467-488

NEWMAN, S.A., (2003) From physics to development: the evolution of morphogenetic mechanism. (to appear in "Origins of Organismal Form", eds. G.B. Müller and S.A. Newman, MIT Press, Cambridge)

SALAZAR-CIUDAD, I., GARCIA-FERNANDEZ, J. and SOLE, R.V. (2000) Gene networks capable of pattern formation: from induction to reaction-diffusion. $J$. Theor. Biol. 205(4) 587-603

SATO, K. ITO, Y., YOMO, T. and KANEKO, K. (2003) On the relation between fluctuation and response in biological systems. Proc. Nat. Acad. Sci. USA. 100 14086-14090

TAKAGI, H. and KANEKO, K. (2005) Dynamical systems basis of metamorphosis: diversity and plasticity of cellular states in reaction diffusion network. J. Theor. Biol. in press

TURING A.M. (1952) The chemical basis of morphogenesis. Phil. Trans. R. Soc. B 237, 37-72

UOCHI, T. and ASASHIMA M. (1996) Sequential gene expression during pronephric tubule formation in vitro in Xenopus ectoderm. Dev. Growth Diff. 38 625634; Private communications.

WOLPERT, L. (1969) Positional information and the spatial pattern of cellular differentiation. J. Theor. Biol. 25, 1-47. 